\title{
Programmes d'assistance humanitaire de la psychothérapie EMDR : traiter les effets psychologiques, physiques et sociétaux des expériences défavorables à travers le monde
}

\author{
Francine Shapiro \\ Mental Research Institute, Palo Alto, California
}

\begin{abstract}
Il est prouvé que les effets négatifs du trauma et d'autres expériences de vie défavorables interfèrent avec le fonctionnement individuel, familial et sociétal. La psychothérapie EMDR (désensibilisation et retraitement par les mouvements oculaires) bénéficie d'un soutien empirique ; elle est recommandée en tant que traitement de première ligne pour le trauma psychologique dans de nombreuses directives de pratique. Elle apporte un traitement à la fois efficace et efficient sans nécessiter de description détaillée de l'événement perturbant ou de tâches à réaliser entre les séances. Ceci permet aux équipes sur le terrain d'offrir une psychothérapie culturellement adaptée lors de journées successives à des personnes dans des régions éloignées et en situation de crise. Des organisations d'aide humanitaire ont mené des projets sur le plan international afin de proposer une psychothérapie EMDR après des catastrophes d'origine naturelle ou humaine et ont contribué à développer des ressources durables en santé mentale à travers le monde. Cette brève introduction apporte une vue d'ensemble des programmes actuels, de la logique qui sous-tend le traitement, ainsi qu'un appel à la mobilisation future.
\end{abstract}

Mots-clés : psychothérapie EMDR ; programmes d'assistance humanitaire ; trauma ; expériences défavorables ; modèle de traitement adaptatif de l'information

$\mathbf{L}$ es articles de ce numéro du Journal of EMDR Practice and Research révèlent l'envergure des programmes d'assistance humanitaire utilisant la psychothérapie EMDR (désensibilisation et retraitement par les mouvements oculaires) et les accomplissements rendus possibles par l'engagement de tous ceux qui ont offert leur temps, leur cour et leurs dons en vue de soulager la souffrance à travers le monde. À l'origine de ces programmes se trouve une réponse à l'attentat d'Oklahoma City en 1995, qui avait dépassé les capacités des intervenants locaux (Shapiro, 2012). Une évaluation de programme a indiqué un taux de réponse au traitement comparable au taux de rémission de $84 \%$ de l'état de stress post-traumatique (ESPT) en trois séances, obtenu dans une étude rigoureuse publiée la même année (Wilson, Becker \& Tinker, 1995).
Par conséquent, les Programmes d'assistance humanitaire (HAP) EMDR sans but lucratif ont été créés en vue d'offrir des services bénévoles en fonction des besoins aux personnes souffrant de trauma lié à des catastrophes d'origine naturelle ou humaine (voir Gelbach, 2014). Des projets ont démarré aux États-Unis et sur le plan international dans des régions comme l'Asie, le Moyen-Orient et l'Amérique latine. L'organisation est maintenant une ONG internationale possédant un statut consultatif auprès du conseil économique et social des Nations Unies et elle a reçu un prix récompensant son excellence clinique, attribué par la Société internationale pour les études sur le stress traumatique (International Society for Traumatic Stress Studies).

L'organisation des États-Unis s'appelle maintenant «Trauma Recovery/EMDR Humanitarian Assistance

This article originally appeared as Shapiro, F. (2014). EMDR Therapy Humanitarian Assistance Programs: Treating the Psychological, Physical, and Societal Effects of Adverse Experiences Worldwide, Journal of EMDR Practice and Research, 8(4), 181-186. Translated by Jenny Ann Rydberg. 
Programs (TR/HAP) » afin de refléter à la fois sa mission internationale et les nombreux réseaux de rétablissement du trauma (Trauma Recovery Networks [TRN]) qui réagissent localement pour venir en aide aux personnes, comme lors de l'attentat du marathon de Boston, des attentats dans des établissements scolaires, des ouragans et des feux de forêt. Une description approfondie du volontariat local impliqué dans deux projets TRN (voir Alter-Reid, Colelli \& Simons, 2014) apporte aux interventions une perspective clinique ainsi que des suggestions pour les projets à venir. De plus, la description des réponses humanitaires à travers le Moyen-Orient, initialement inaugurées par une formation HAP en Palestine en 2005 (voir Zaghrout-Hodali, 2014), donne vie à l'importance d'un traitement efficace pour les rescapés de violences politiques, de détention et de torture, avec des descriptions cliniques des personnes traitées dans un camp de réfugiés en Palestine, dans un camp de réfugiés syrien en Jordanie et dans une zone de combat en Lybie.

Au cours des deux décennies suivant la réponse initiale à Oklahoma, le développement de formations à la psychothérapie EMDR à travers le monde s'accompagne de la création d'associations humanitaires extérieures aux États-Unis. Le programme d'assistance humanitaire européen (HAP Europe ; voir Farrell, 2014), des associations EMDR nationales en Europe (voir Fernandez, Callerame, Maslovaric \& Wheeler, 2014) et le programme d'assistance humanitaire " Trauma Aid » en Allemagne (voir Mattheß \& Sodemann, 2014) ont apporté de l'aide tant localement qu'à l'extérieur de l'Europe. Depuis 2007, malgré de nombreuses difficultés, des formations à la psychothérapie EMDR ont été organisées à travers l'Afrique par TR/HAP, Trauma Aid, HAP Europe et des membres de diverses associations EMDR nationales européennes (voir Zimmermann, 2014). En Asie, la formation à la psychothérapie EMDR et le traitement EMDR ont débuté en 1998 en réponse aux appels à l'aide après diverses catastrophes naturelles. En particulier, de nombreuses associations nationales et régionales ont été créées en Asie à la suite des projets humanitaires et des efforts des organisations américaines et européennes. En 2010, nous avons accueilli la création d'EMDR Asie. Ses membres ont aidé des milliers de personnes tant par la formation que par les réponses post-catastrophe (voir Mehrotra, 2014). En 1998, des membres d’EMDR HAP aux États-Unis ont répondu à une demande de coopération après un ouragan au Mexique. À la suite de ce projet, l'Association mexicaine pour le soutien en santé mentale lors de crises, la Fondation latino-américaine et caribéenne pour la recherche sur le trauma psychologique ainsi que des organisations HAP de l'Argentine et du Brésil ont fourni une aide humanitaire considérable à travers l'Amérique latine et les Caraïbes (voir Jarero, Artigas, Uribe \& Miranda, 2014).

Les membres de toutes ces organisations ont, bénévolement, animé des formations à la psychothérapie EMDR et apporté un traitement EMDR à des populations démunies à travers le monde. Par conséquent, l'applicabilité interculturelle de la psychothérapie EMDR est clairement établie avec des projets fructueux à travers l'Afrique, l'Asie, les Caraíbes, l'Europe, l'Amérique latine, le Moyen-Orient et les États-Unis. Les évaluations de projet ont démontré des effets positifs pour les protocoles tant individuels que de groupe (p. ex. Aduriz, Bluthgen \& Knopfler, 2009 ; Fernandez, Gallinari \& Lorenzetti, 2004 ; Jarero, Artigas \& Hartung, 2006 ; Konuk et coll., 2006 ; Silver, Rogers, Knipe \& Colelli, 2005 ; Zaghrout-Hodali, Alissa \& Dodgson, 2008 ; voir aussi Farrell, 2014 ; Jarero et coll., 2014). En plus du traitement de crise, l'accent est placé sur le développement de ressources de traitement en santé mentale avec les services locaux. Tous les projets sont des collaborations sur invitation et sont très attentifs aux différences culturelles et aux besoins psychosociaux.

\section{Psychothérapie EMDR}

Bien qu'elle ait été initialement considérée avec scepticisme, la psychothérapie EMDR a été validée par plus de vingt-quatre essais randomisés contrôlés (voir http:/ / www.emdrhap.org/ content/ what-is-emdr/ research-findings/) et de nombreuses méta-analyses (p. ex. Bisson, Roberts, Andrew, Cooper \& Lewis, 2013; Watts et coll., 2013). Vingt autres essais randomisés et une méta-analyse récente (Lee \& Cuijpers, 2013) ont montré les effets positifs des mouvements oculaires, comprenant la diminution rapide de la détresse émotionnelle. Il importe de noter que la psychothérapie EMDR a été recommandée en tant que traitement du trauma efficace validé par toute une palette d'organisations, tant aux États-Unis (p. ex. Department of Veterans Affairs \& Department of Defense, 2010 ; Substance Abuse and Mental Health Services Administration, National Registry of Evidence-Based Programs and Practices, 2011) que sur le plan international (p. ex. International Society for Traumatic Stress Studies ; Foa, Keane, Friedman \& Cohen, 2009). Selon les récentes Directives pour la gestion des affections spécifiquement liées aux stress [Guidelines for the Management of Conditions Specifically Related to Stress] de l’Organisation mondiale de la santé (WHO ; 2013), 
la thérapie cognitive comportementale centrée sur le trauma (TCC) et la psychothérapie EMDR sont les seules psychothérapies recommandées pour les enfants, les adolescents et les adultes atteints d'un ESPT. Comme les directives de l'OMS l'indiquent:

La thérapie [EMDR] est fondée sur l'idée que les pensées, émotions, sensations et comportements négatifs sont le résultat de souvenirs non retraités. Le traitement implique les procédures standard qui comprennent le fait de se centrer simultanément sur (a) des associations spontanées d'images, de pensées, d'émotions et de sensations corporelles et (b) une stimulation bilatérale qui prend le plus souvent la forme de mouvements oculaires répétés. Comme la TCC centrée sur le trauma, l'EMDR vise à réduire la détresse subjective et à renforcer les cognitions adaptatives liées à l'événement traumatique. Contrairement à la TCC centrée sur le trauma, l'EMDR n'implique pas (a) de description détaillée de l'événement, (b) de remise en question directe des croyances, (c) d'exposition étendue ou (d) de tâche à réaliser entre les séances. (p. 1)

La description des directives de pratique de l'OMS met en avant des implications importantes pour le traitement humanitaire. Comme la psychothérapie EMDR ne nécessite pas de tâche à réaliser entre les séances, le traitement peut être administré lors de journées consécutives, ce qui signifie que les équipes sur le terrain peuvent apporter un soulagement à la fois efficace et efficient aux personnes qui en ont besoin, même dans des régions éloignées. Puisque des descriptions détaillées de l'événement ne sont pas nécessaires, la psychothérapie s'adapte aux membres de sociétés réticentes à la divulgation et aux personnes qui autrement hésiteraient à participer en raison de violences sexuelles ou d'expériences de guerre qui ont provoqué de la culpabilité ou de la honte. Comme l'indique le modèle de traitement adaptatif de l'information (TAI) (Shapiro, 1995, 2001, 2014 ; Solomon \& Shapiro, 2008) qui guide les applications cliniques de la psychothérapie EMDR, les souvenirs non retraités d'événements négatifs contiennent les émotions, les sensations physiques et les croyances vécues lors de l'incident. Ces souvenirs peuvent être déclenchés par des situations actuelles, provoquant l'émergence de perceptions antérieures encodées qui influencent les réactions présentes de l'individu. Reconnaître que les expériences de vie défavorables sont à l'origine de toute une gamme de pathologies et d'autres effets invalidants, c'est souligner l'urgence de traiter les victimes de traumas et d'autres événements perturbants.
Ces souvenirs non retraités d'expériences défavorables s'entendent comme affectant la santé tant mentale que physique mais aussi les capacités d'apprentissage et la qualité des relations personnelles et professionnelles. De plus, les séquelles du trauma peuvent se transmettre entre les générations, produisant des cycles continus de violence et de souffrance qui touchent les individus, les familles et les sociétés.

\section{Effets interpersonnels du trauma non retraité}

Comme le modèle TAI l'indique, les traumas et autres expériences de vie défavorables non retraités ont des effets individuels et interpersonnels profonds (Shapiro, 1995, 2001, 2014). La recherche a révélé des conséquences graves et persistantes tout au long de la vie. Au vu de la prévalence de la traumatisation générée à travers le monde par les violences directes, naturelles, structurelles et culturelles (voir Carriere, 2014), le besoin d'un traitement du trauma rapide est clair. Les effets intergénérationnels comprennent un défaut de liens affectifs et un attachement perturbé en raison de la colère, de la dépression, de l'anxiété et de la crainte inhérentes au trouble. Sans surprise, la recherche a montré que les mères atteintes d'un ESPT courent plus de risques de maltraiter leurs enfants (Chemtob, Gudiño \& Laraque, 2013). Les implications en sont profondes lorsque l'on considère la recherche démontrant que " les châtiments corporels sévères [c.-à-d., pousser, empoigner, bousculer, gifler, frapper] en l'absence de maltraitance infantile [plus sévère] sont associés à des troubles de l'humeur, à des troubles anxieux, à la dépendance aux stupéfiants et à l'alcool et aux troubles de la personnalité dans un échantillon de la population générale " (Afifi, Mota, Dasiewicz, MacMillan \& Sareen, 2012, p. 1). D’autres recherches ont révélé une corrélation positive entre diverses formes de maltraitance et de symptômes psychotiques chez les enfants (Arseneault et coll., 2011 ; Varese et coll., 2012). La recherche a également indiqué que la "discordance au niveau de la maladie psychotique entre individus de la même famille peut s'expliquer par l'exposition différentielle au trauma " (Heins et coll., 2011, p. 1286). En somme, bien que des facteurs génétiques prédisposants puissent être impliqués, " contrairement aux croyances traditionnelles chez les chercheurs et cliniciens à orientation biologique, l'étiologie de la psychose et de la schizophrénie a un fondement tout aussi social [p. ex. l'adversité précoce] que les problèmes de santé mentale non psychotiques tels que l'anxiété et la dépression (Read, Fosse, Moskowitz \& Perry, 2014, p. 73). Compte tenu 
des déficiences générales et du potentiel d'agression et de violence dans cette population (Douglas, Guy $\&$ Hart, 2009), les risques individuels, familiaux et sociétaux sont clairs. Toutes ces recherches démontrent le besoin de traitements efficaces et efficients tels que la psychothérapie EMDR pour répondre à la traumatisation présente et intergénérationnelle. Heureusement, des recherches préliminaires ont montré que la psychothérapie EMDR réduit tant l'ESPT que les hallucinations auditives chez les personnes souffrant de psychose (Van den Berg \& Van der Gaag, 2012).

Le besoin d'un traitement rapide et efficace devient encore plus pressant lorsque l'on considère les résultats de l'étude sur les expériences défavorables de l'enfance (Adverse Childhood Experiences [ACE]) qui a évalué plus de 17000 participants et « trouvé une forte relation dose-réponse entre l'envergure de l'exposition à des violences ou à un dysfonctionnement familial dans l'enfance et de multiples facteurs de risque pour plusieurs des premières causes de mortalité chez les adultes (Felitti et coll., 1998, p. 251). Ces risques de mortalité comprennent des affections physiquement invalidantes comme la cardiopathie ischémique, le cancer, la pneumopathie chronique, les fractures osseuses et les maladies du foie. Le fait que la traumatisation dans l'enfance puisse avoir des effets mentaux et physiques qui perdurent tout au long de la vie devrait constituer une incitation importante à mettre en lumière les crises de santé globales auxquelles nous sommes tous confrontés et à commencer à apporter une réponse complète.

\section{Effets sociétaux des souvenirs non retraités}

Ainsi que l'article de Carriere (2014) l'indique, il est vital qu'une attention et des ressources suffisantes soient fournies pour que le traitement puisse être offert à des millions de personnes atteintes à travers le monde. Les effets délétères du trauma non traité comportent des implications sociétales graves. Bien que de nombreuses organisations se consacrent à offrir des services pour venir en aide aux pays à faibles revenus dans leur développement et pour atteindre des buts en termes d'éducation, des problèmes de santé mentale non traités peuvent sérieusement freiner leur succès. Par exemple, les effets rapportés du trauma et d'autres expériences défavorables comprennent également l'alcoolisme, la toxicomanie et la dépression (Felitti et coll., 1998), ce qui peut de toute évidence empêcher les personnes dans des régions sujettes aux crises de profiter pleinement des opportunités de développement (p. ex. emplois, formations, services sanitaires) proposées par divers organismes des Nations unies, de la société civile, d'organismes caritatifs et d'entreprises. Les stratégies de coping liées aux addictions et au trauma interfèrent avec les motivations positives et alimentent la colère et l'agressivité. Celles-ci ont à leur tour des implications sociétales importantes car une traumatisation largement répandue et non soulagée peut engendrer à la fois le dysfonctionnement familial et une culture omniprésente de méfiance et de discorde.

Dans la perspective du modèle TAI, les souvenirs non retraités ont également contribué à des générations de violences continues alimentées par la méfiance, la colère, la douleur, la peur et l'hypervigilance inhérentes à la traumatisation. En plus de ces effets qui atteignent les individus les plus impliqués, la narration répétée des humiliations et agressions physiques antérieures peut causer une traumatisation secondaire chez les générations suivantes (p. ex. Bombay, Matheson \& Anisman, 2013). Les enfants ne sont pas seulement affectés par la détresse de leurs parents, mais en entendant de telles histoires, ils peuvent s'imaginer comme ayant vécu le trauma historique et réagir en conséquence. Tel qu'indiqué par le modèle TAI, tout rappel subséquent du trauma peut déclencher les émotions négatives, sensations physiques et croyances créées par l'événement et influencer la perception du présent. Ceci peut produire un antagonisme continu envers " les autres »- des groupes définis par l'ethnie, la religion, la race, la classe sociale ou la caste - désignés comme adversaires en raison du trauma historique (p. ex. Bombay, Matheson \& Anisman, 2014). Les tentatives d'agir comme médiateur pour trouver des solutions pacifiques peuvent également se trouver entravées par ces réactions automatiques puisque l'appréciation des personnes « autour de la table » est influencée par la colère, la peur, l'humiliation et la honte instillées par les expériences passées. De plus, les médiateurs, les prestataires de services, les intervenants en aide humanitaire et les casques bleus peuvent être psychologiquement diminués en raison de la traumatisation secondaire par les descriptions détaillées d'événements passés. Comme de nombreuses études l'ont montré, les effets automatiques de traumas non résolus peuvent se résoudre par les protocoles standard de la psychothérapie EMDR (Shapiro, 2001), qui ciblent (a) les événements passés, (b) les situations actuelles qui déclenchent des réponses négatives et (c) les compétences nécessaires à un fonctionnement adaptatif futur.

Il importe de noter que la recherche a également révélé que les personnes atteintes d'ESPT éprouvent des difficultés à prendre du recul par rapport aux indices de menace (Pineles, Shipherd, Mostoufi, Abramovitz 
\& Yovel, 2009), ce qui encombrerait certainement les personnes tentant de promouvoir des solutions positives. Heureusement, une recherche préliminaire (El Khoury-Malhame et coll., 2011) a indiqué que la psychothérapie EMDR peut mener à une normalisation et à l'élimination du biais attentionnel négatif. Bien que d'autres recherches soient nécessaires, ces résultats indiquent que la disponibilité d'un traitement du trauma rapide et efficace pourrait aider à créer la réconciliation, une coexistence pacifique et le potentiel d'un développement non violent. Ceci souligne d'autant plus le besoin d'accroître les efforts pour apporter des services en santé mentale qui répondent aux effets invalidants du trauma à travers des opportunités de soin étendues, des efforts de collaboration globale et un soutien à la recherche (Carriere, 2014).

\section{Un engagement à agir}

Au cours des deux dernières décennies, les bénévoles dans les divers programmes d'assistance humanitaire EMDR se sont dévoués à l'allègement de la souffrance dans des populations mal desservies à travers le monde. Ils ont reconnu que la peur, la dépression, l'anxiété, la colère et la douleur des expériences traumatiques non retraitées ont des effets invalidants sur l'individu qui peuvent faire dérailler tout espoir d'une vie heureuse et productive. Ils reconnaissent également l'impact négatif sur les familles, puisque la douleur de l'individu peut conduire à des violences domestiques et à la transmission intergénérationnelle du dysfonctionnement à travers des liens affectifs inadéquats, des agressions ou le désengagement. Cette prise de conscience les a motivés à atteindre les personnes situées dans des endroits peu desservis à travers le monde, tant dans leur pays d'origine qu'ailleurs. Et enfin, ils ont reconnu l'impact sociétal, comprenant la douleur incessante causée par les violences ethnopolitiques répétées génération après génération. Cela les a motivés à offrir la formation à l'EMDR et un traitement EMDR dans des lieux tels que l'Irlande du nord, les Balkans et le Moyen-Orient, dans l'espoir de contribuer à la réconciliation et à la paix.

Ces bénévoles dévoués reconnaissent que le trauma peut être traité de manière efficace et efficiente, et ils se sont consacrés à apporter la guérison à ceux qui en ont besoin. Ils ont continué inlassablement, déterminés que personne ne soit laissé pour compte. Ils reconnaissent qu'un traitement de santé mentale efficace devrait être à la portée de tous et non seulement dans les régions plus aisées des pays développés. Nous leur rendons hommage ainsi qu'à ceux qui ont soutenu les projets par leurs dons et à ceux qui ont aidé à ouvrir des portes permettant une intervention rapide en établissant l'efficacité de la psychothérapie EMDR grâce à leur dévouement à une recherche rigoureuse. Cette édition anniversaire du Journal of EMDR Practice and Research offre aux lecteurs une vue d'ensemble de la guérison accomplie à travers ces projets humanitaires et une incitation à faire le nécessaire pour étendre la portée de ces efforts aux millions de personnes qui souffrent encore inutilement à travers le monde.

\section{Bibliographie}

Aduriz, M. E., Bluthgen, C., \& Knopfler, C. (2009). Helping child flood victims using group EMDR intervention in Argentina: Treatment outcome and gender differences. International Journal of Stress Management, 16, 138-153.

Afifi, T. O., Mota, N. P., Dasiewicz, P., MacMillan, H. L., \& Sareen, J. (2012). Physical punishment and mental disorders: Results from a nationally representative US sample. Pediatrics, 130(2), 184-192. http:/ / www.dx.doi .org/10.1542/ peds.2011-2947

Alter-Reid, K., Colelli, G., \& Simons, N. (2014). When disaster strikes our local communities: U.S. EMDR trauma recovery network coordinators reflect on lessons learned. Journal of EMDR Practice and Research, 8(4), 205-214.

Arseneault, L., Cannon, M., Fisher, H. L., Polanczyk, G., Moffitt, T. E., \& Caspi, A. (2011). Childhood trauma and children's emerging psychotic symptoms: A genetically sensitive longitudinal cohort study. Am J Psychiatry, 168, 65-72.

Bisson, J., Roberts, N. P., Andrew, M., Cooper, R., \& Lewis, C. (2013). Psychological therapies for chronic post-traumatic stress disorder (PTSD) in adults. Cochrane Database of Systematic Reviews, (12), CD003388. http: / / www .dx.doi.org/10.1002/14651858.CD003388.pub4

Bombay, A., Matheson, K., \& Anisman, H. (2013). The intergenerational effects of Indian residential schools: Implications for the concept of historical trauma. Transcultural Psychiatry, 51(3), 320-338.

Bombay, A., Matheson, K., \& Anisman, H. (2014). Appraisals of discriminatory events among adult offspring of Indian residential school survivors: The influences of identity centrality and past perceptions of discrimination. Cultural Diversity and Ethnic Minority Psychology, 20, 75-86.

Carriere, R. (2014). Scaling up what works: Using EMDR to help confront the world's burden of traumatic stress. Journal of EMDR Practice and Research, 8(4), 187-195.

Chemtob, C. M., Gudiño, O. G., \& Laraque, D. (2013). Maternal posttraumatic stress disorder and depression in pediatric primary care: Association with child maltreatment and frequency of child exposure to traumatic events. JAMA Pediatrics, 167(11), 1011-1018.

Department of Veterans Affairs \& Department of Defense (2010). VA/DoD clinical practice guidelines for the 
management of posttraumatic stress. Washington, DC: Veterans Health Administration, Department of Veterans Affairs and Health Affairs, Department of Defense.

Douglas, K. S., Guy, L. S., \& Hart, S. D. (2009). Psychosis as a risk factor for violence to others: A meta-analysis. Psychological Bulletin, 135(5), 679-706.

El Khoury-Malhame, M., Lenteaume, L., Beetz, E. M., Rogues, J., Reynaud, E., Samuelian, J. C., . . Khalfa, S. (2011). Attentional bias in post-traumatic stress disorder diminishes after symptom amelioration. Behaviour Research and Therapy, 49(11), 796-801.

Farrell, D. (2014). Developing EMDR therapy in Pakistan as part of a humanitarian endeavour. Journal of EMDR Practice and Research, 8(4), 233-239.

Felitti, V. J., Anda, R. F., Nordenberg, D., Williamson, D. F., Spitz, A. M., Edwards, V., . . . Marks, J. S. (1998). Relationship of childhood abuse and household dysfunction to many of the leading causes of death in adults: The adverse childhood experiences (ACE) study. American Journal of Preventive Medicine, 14(4), 245-258.

Fernandez, I., Callerame, C., Maslovaric, G., \& Wheeler, K. (2014). EMDR Europe humanitarian programs: Development, current status, and future challenges. Journal of EMDR Practice and Research, 8(4), 215-224.

Fernandez, I., Gallinari, E., \& Lorenzetti, A. (2004). A school-based EMDR intervention for children who witnessed the Pirelli building airplane crash in Milan, Italy. Journal of Brief Therapy, 2, 129-136.

Foa, E. B., Keane, T. M., Friedman, M. J., \& Cohen, J. A. (Eds.). (2009). Effective treatments for PTSD: Practice guidelines from the International Society for Traumatic Stress Studies (2nd ed.). New York, NY: Guilford Press.

Gelbach, R. (2014). EMDR humanitarian assistance programs: Twenty years and counting. Journal of EMDR Practice and Research, 8(4), 196-204.

Heins, M., Simons, C., Lataster, T., Pfeifer, S., Vermissen, D., Lardinois, M., . . . Myin-Germeys, I. (2011). Childhood trauma and psychosis: A case-control and casesibling comparison across different levels of genetic liability, psychopathology, and type of trauma. Am J Psychiatry, 168(12), 1286-1294.

Jarero, I., Artigas, L., \& Hartung, J. (2006). EMDR integrative group treatment protocol: A post-disaster trauma intervention for children and adults. Traumatology, 12, 121-129.

Jarero, I., Artigas, L., Uribe, S., \& Miranda, A. (2014). EMDR therapy humanitarian trauma recovery interventions in Latin America and the Caribbean. Journal of EMDR Practice and Research, 8(4), 260-268.

Konuk, E., Knipe, J., Eke, I., Yuksek, H., Yurtsever, A., \& Ostep, S. (2006). The effects of EMDR therapy on post-traumatic stress disorder in survivors of the 1999 Marmara, Turkey earthquake. International Journal of Stress Management, 13, 291-308.

Lee, C. W., \& Cuijpers, P. (2013). A meta-analysis of the contribution of eye movements in processing emotional memories. Journal of Behavior Therapy and Experimental Psychiatry, 44, 231-239.

Mattheß, H., \& Sodemann, U. (2014). Trauma-Aid, Humanitarian Assistance Program Germany. Journal of EMDR Practice and Research, 8(4), 225-232.

Mehrotra, S. (2014). Humanitarian projects and growth of EMDR therapy in Asia. Journal of EMDR Practice and Research, 8(4), 252-259.

Pineles, S. L., Shipherd, J. C., Mostoufi, S. M., Abramovitz, S. M., \& Yovel, I. (2009). Attentional biases in PTSD: More evidence for interference. Behaviour Research and Therapy, 47(12), 1050-1057.

Read, J., Fosse, R., Moskowitz, A., \& Perry, B. (2014). The traumagenic neurodevelopmental model of psychosis revisited. Neuropsychiatry, 4(1), 65-79.

Shapiro, F. (1995). Eye movement desensitization and reprocessing: Basic principles, protocols, and procedures. New York, NY: Guilford Press.

Shapiro, F. (2001). Eye movement desensitization and reprocessing: Basic principles, protocols, and procedures (2nd ed.). New York, NY: Guilford Press.

Shapiro, F. (2012). EMDR humanitarian assistance programs: Building sustainable mental health resources worldwide. Stress Points, 12, 2-3.

Shapiro, F. (2014). The role of eye movement desensitization and reprocessing (EMDR) therapy in medicine: Addressing the psychological and physical symptoms stemming from adverse life experiences. The Permanente Journal, 18, 71-77.

Silver, S. M., Rogers, S., Knipe, J., \& Colelli, G. (2005). EMDR therapy following the 9/11 terrorist attacks: A community-based intervention project in New York City. International Journal of Stress Management, 12, 29-42.

Solomon, R. W., \& Shapiro, F. (2008). EMDR and the adaptive information processing model: Potential mechanisms of change. Journal of EMDR Practice and Research, 2, 315-325.

Substance Abuse and Mental Health Services Administration, National Registry of Evidence-Based Programs and Practices. (2011). Eye movement desensitization and reprocessing. Retrieved from http://nrepp.samhsa.gov/ ViewIntervention. aspx?id=199

Van den Berg, D. P. G., \& Van der Gaag, M. (2012). Treating trauma in psychosis with EMDR: A pilot study. Journal of Behavior Therapy and Experimental Psychiatry, 43, 664-671.

Varese, F., Smeets, F., Drukker, M., Lieverse, R., Lataster, T., Viechtbauer, W., . . . Bentall, R. P. (2012). Childhood adversities increase the risk of psychosis: A meta-analysis of patient-control, prospective- and cross-sectional cohort studies. Schizophrenia Bulletin, 38(4), 661-671.

Watts, B. V., Schnurr, P. P., Mayo, L., Young-Xu, Y., Weeks, W. B., \& Friedman, M. J. (2013). Meta-analysis of the efficacy of treatments for posttraumatic stress disorder. Journal of Clinical Psychiatry, 74(6), e541-e550. http:// dx.doi.org/10.4088/JCP.12r08225 
Wilson, S., Becker, L. A., \& Tinker, R. H. (1995). Eye movement desensitization and reprocessing (EMDR): Treatment for psychologically traumatized individuals. Journal of Consulting and Clinical Psychology, 63, 928-937.

World Health Organization (2013). Guidelines for the management of conditions specifically related to stress. Geneva, Switzerland: Author.

Zaghrout-Hodali, M. (2014). Humanitarian work using EMDR in Palestine and the Arab world. Journal of EMDR Practice and Research, 8(4), 248-251.
Zaghrout-Hodali, M., Alissa, F., \& Dodgson, P. W. (2008). Building resilience and dismantling fear: EMDR group protocol with children in an area of ongoing trauma. Journal of EMDR Practice and Research, 2, 106-113.

Zimmermann, E. (2014). EMDR humanitarian work: Providing trainings in EMDR therapy to African clinicians. Journal of EMDR Practice and Research, 8(4), 240-247.

Merci d'adresser toute correspondance concernant cet article à : Francine Shapiro, $\mathrm{PhD}, \mathrm{PO} 750$, Watsonville, CA 95077, États-Unis. Courriel : fshapiro@mcn.org 